\title{
Chronic Augmentation of Endocannabinoid Levels Persistently Increases Dopaminergic Encoding of Reward Cost and Motivation
}

\author{
Dan P. Covey, ${ }^{1}$ Edith Hernandez, ${ }^{2}$ Miguel Á. Luján, ${ }^{3}$ and Joseph F. Cheer ${ }^{3,4}$ \\ ${ }^{1}$ Department of Neuroscience, Lovelace Biomedical Research Institute, Albuquerque, New Mexico 87108, ${ }^{2}$ Medical Scientist Training Program (MD/ \\ $\mathrm{PhD}$ ), University of Minnesota Medical School, Minneapolis, Minnesota 55455, ${ }^{3}$ Department of Anatomy and Neurobiology, University of Maryland \\ School of Medicine, Baltimore, Maryland 21201, and ${ }^{4}$ Department of Psychiatry, University of Maryland School of Medicine, Baltimore, Maryland
} 21201

Motivational deficits characterized by an unwillingness to overcome effortful costs are a common feature of neuropsychiatric and neurologic disorders that are insufficiently understood and treated. Dopamine (DA) signaling in the nucleus accumbens (NAc) facilitates goal-seeking, but how NAc DA release encodes motivationally salient stimuli to influence effortful investment is not clear. Using fast-scan cyclic voltammetry in male and female mice, we find that NAc DA release diametrically responds to cues signaling increasing cost of reward, while DA release to the reward itself is unaffected by its cost. Because endocannabinoid (eCB) signaling facilitates goal seeking and NAc DA release, we further investigated whether repeated augmentation of the eCB 2-arachidonoylglycerol with a low dose of a monoacylglycerol lipase (MAGL) inhibitor facilitates motivation and DA signaling without the development of tolerance. We find that chronic MAGL treatment stably facilitates goal seeking and DA encoding of prior reward cost, providing critical insight into the neurobiological mechanisms of a viable treatment for motivational deficits.

Key words: dopamine; endocannabinoids; motivation; nucleus accumbens; voltammetry

\section{Significance Statement}

Decades of work has established a fundamental role for dopamine neurotransmission in motivated behavior and cue-reward learning, but how dopaminergic encoding of cues associates with motivated action has remained unclear. Specifically, how dopamine neurons signal future and prior reward cost, and whether this can be modified to influence motivational set points is not known. The current study provides important insight into how dopamine neurons encode motivationally relevant stimuli to influence goal-directed action and supports cannabinoid-based therapies for treatment of motivational disorders.

\section{Introduction}

Dopamine (DA) release in the nucleus accumbens (NAc) controls the propensity to overcome effortful costs during reward seeking (Salamone and Correa, 2012). Accumbal DA release is proposed to signal reward "value" by differentially responding to rewards and their associated cues according to expected and experienced reward magnitude or predictability (Schultz, 2002, 2015). Yet, value is ultimately defined by how much a consumer is willing to pay for a good or service in units of currency. But

\footnotetext{
Received Feb. 5, 2021; revised June 16, 2021; accepted June 21, 2021.

Author contributions: D.P.C., E.H., and J.F.C. designed research; D.P.C. and E.H. performed research; D.P.C., E.H., and M.Á.L. analyzed data; D.P.C. wrote the paper.

This work was funded by National Institutes of Health | National Institute on Drug Abuse Grants 4R00-DA047432-03 (D.P.C.) and 2R01-DA-022340-06A1 (J.F.C.).

The authors declare no competing financial interests.

Correspondence should be addressed to Joseph F. Cheer at jcheer@som.umaryland.edu.

https://doi.org/10.1523/JNEUROSCI.0285-21.2021

Copyright $\odot 2021$ the authors
}

how DA release in the NAc signals the willingness to pay for rewards is unclear (Walton and Bouret, 2019). Notably, a reluctance to overcome effortful costs (i.e., apathy) is a common feature of multiple psychiatric and neurologic disorders that affect DA function, including Parkinson's and Huntington's diseases, depression, schizophrenia, and multiple sclerosis (Covey et al., 2016; Le Heron et al., 2019). Yet motivational deficits that severely impact daily functioning and quality of life for numerous patients and their caregivers remain insufficiently understood and treated.

The endocannabinoid (eCB) system controls goal-directed behavior and DA neuron function, making it a promising target for pharmacotherapies aimed at treating motivational disorders (Covey et al., 2016; Peters et al., 2021). The eCB 2-arachidonoylglycerol (2-AG) orchestrates DA neuron excitability via terminal regulation of ventral tegmental area (VTA) inputs (Sagheddu et al., 2015). 2-AG binding onto the cannabinoid type 1 (CB1) receptor in the VTA increases DA neuronal firing and DA release 
in the NAc, and facilitates reward seeking (Cheer et al., 2007; Oleson et al., 2012; Wang et al., 2015; Covey et al., 2018). But whether targeting 2-AG signaling to facilitate DA function and motivated behavior represents a viable, long-term treatment option is unclear. First, chronic inhibition of 2-AG degradation using the monoacylglycerol lipase (MAGL) inhibitor JZL184 (JZL) can produce rapid CB1 receptor desensitization and behavioral tolerance (Baggelaar et al., 2018). Second, how eCB manipulations influence goal-directed behavior by shaping DAergic encoding of value-related stimuli is yet to be determined.

Here, we trained mice on a trial-based, chain schedule of reinforcement during which reinforcer cost increased on each trial according to a progressive ratio (PR) schedule, and recorded subsecond accumbal DA dynamics in response to cues signaling reward cost and reward receipt. We further investigated how chronic augmentation of 2-AG signaling with JZL influences dopaminergic encoding of value-related stimuli. We find that cue-evoked DA release in the NAc diametrically and preferentially encodes upcoming and experienced cost across the PR session, but not reward receipt. Moreover, chronic augmentation of 2-AG stably invigorates effortful responding and potentiates DAergic encoding of reward cost across multiple sessions, suggesting a viable treatment option for a neurobiological substrate of motivational deficits.

\section{Materials and Methods}

Animals. Male $(n=14)$ and female $(n=16)$ C57BL/6 mice (weight, 20-30 g) were received from The Jackson Laboratory and housed in a temperature-controlled room maintained on a reverse $12 \mathrm{~h} \mathrm{light/dark}$ cycle (7:00 A.M. to 7:00 P.M.). Following surgery, mice were group housed and allowed ad libitum access to water and food. Mice were then restricted to $85-90 \%$ body weight for the duration of behavioral measures. All experiments were conducted in the light cycle of the animal. Animal care and experimental procedures conformed to the National Institutes of Health Guide for the Care and Use of Laboratory Animals and were approved by the Institutional Animal Use and Care Committee at the University of Maryland, Baltimore.

Surgery. Mice were anesthetized with isoflurane in $\mathrm{O}_{2}$ (4\% induction and $1 \%$ maintenance) and implanted with a chronic voltammetry electrode targeting the NAc (relative to bregma: anteroposterior, +1.2 AP; mediolateral, +1.1; dorsoventral, -3.7) and a $\mathrm{Ag} / \mathrm{AgCl}$ reference electrode in the contralateral superficial cortex. All components were permanently affixed with dental cement (Grip Cement Dentsply).

In vivo fast-scan cyclic voltammetry. Fast-scan cyclic voltammetry (FSCV) was used to monitor DA concentration changes by applying a triangular waveform $(-0.4$ to $+1.3 \mathrm{~V}$ at $400 \mathrm{~V} / \mathrm{s})$ at $10 \mathrm{~Hz}$ to implanted carbon fiber microelectrodes. FSCV is able to extract changes in faradaic current because of redox reactions at the carbon fiber surface, and this change in current is proportional to the concentration of electroactive analytes. Here, we report all FSCV measures in current (in nanoamperes) as this is the unit of measurement that serves as an estimate of the change in concentration. Principal component regression analysis was used to statistically extract the DA component from the voltammetric recording during the $3 \mathrm{~s}$ period following stimulus onset (see below; Heien et al., 2005). Training sets were created using noncontingent optogenetically evoked DA signals obtained following a recording session and a standard set of five basic $\mathrm{pH}$ shift voltammograms.

Apparatus. Mice were tested in operant chambers $(21.6 \times 17.6 \times$ $14 \mathrm{~cm}$; Med Associates) housed within sound-attenuating enclosures. Each chamber was equipped with two retractable levers (located $2 \mathrm{~cm}$ above the floor) and one LED stimulus light located above each lever $(4.6 \mathrm{~cm}$ above the lever). An external food magazine delivered sucrose pellets (14 mg; Bio-Serv) to a dispenser centrally located between the two levers. A houselight and a white-noise speaker $(80 \mathrm{~dB}$, masking noise background) were located on the opposite wall.

Behavior. Two weeks following surgery, mice were trained to lever press for sucrose pellet reinforcement on a trial-based chain schedule of reinforcement (Fig. 1). The first group of mice $(n=6$ males, $n=5$ females) were used to validate the behavioral task and did not receive drug treatment. The second group of mice ( $n=8$ male, $n=11$ female) received JZL-184 and vehicle (VEH) treatment. All mice were trained in the same manner. The behavioral task consisted of the following two phases: (1) on reaching the response requirement on one lever ["seeking" lever $\left(\operatorname{Lev}_{\mathrm{S}}\right)$ ]; and (2) a single press on the opposite lever ["taking" lever $\left.\left(\mathrm{Lev}_{\mathrm{T}}\right)\right]$ delivered a single sucrose pellet. Training began with $30 \mathrm{~min}$ sessions of a fixed-ratio one (FR1) schedule on $\mathrm{Lev}_{\mathrm{T}}$. FR1 session onset was signaled by illumination of the cue light ["taking" cue $\left(\mathrm{Cue}_{\mathrm{T}}\right)$ ] above $\mathrm{Lev}_{\mathrm{T}}$ and presentation of a tone (white noise or $2.5 \mathrm{kHz}$ tone, counterbalanced), followed 3-6 s later by the extension of both levers. For each event, stimulus-entrained DA release was quantified during the first $3 \mathrm{~s}$ following stimulus presentation. Following a correct response, a single sucrose pellet was delivered, both levers were retracted, and $\mathrm{Cue}_{\mathrm{T}}$ turned off for a variable timeout period (30-90 s) before the next trial began. In all subsequent sessions, the response requirement on $\mathrm{Lev}_{\mathrm{T}}$ remained at one. After stable responding ( $>80 \%$ active presses and less than five trial omissions, typically between 5 and 10 sessions), mice proceeded to the next stage during which trial onset was signaled by illumination of the cue light over the opposite lever $\left(\mathrm{Lev}_{\mathrm{S}}\right)$ and presentation of the opposite tone [seeking cue $\left(\mathrm{Cue}_{\mathrm{S}}\right)$ ], followed 3-6 s later by extension of Levs. A single response on the seeking lever led to retraction of both levers, Cue $e_{S}$ was turned off, and $\mathrm{Cue}_{\mathrm{T}}$ was presented, followed 3-6 s later by extension of the $\operatorname{Lev}_{\mathrm{T}}$. One correct response led to the delivery of a single sucrose pellet, lever retraction, and $\mathrm{Cue}_{\mathrm{T}}$ turned off for a variable timeout period ( $30-90 \mathrm{~s}$ ) before the next trial began. Following stable responding ( $>90 \%$ seeking lever presses and less than two trial omissions for two consecutive sessions, typically between two and eight sessions), the response requirement on the $\mathrm{Lev}_{\mathrm{S}}$ increased to an FR5 schedule for one session, after which the response requirement switched to a PR schedule. During the PR schedule, the number of lever presses required to earn each reward (i.e., response ratio) increased exponentially across successive trials [response ratio $\left.=\left(5 \times \mathrm{e}^{(0.2 \times \text { reward number })}-5\right)\right]$, yielding ratios of, for example, $1,2,4,6,9,12,15,20,25,32,40,50,62,77,95$, and 118 after rounding to the closest integer (Hodos, 1961; Covey et al., 2018). The final ratio attained (i.e., break point) is considered a metric of inherent motivation to expel effort to gain reward. Sessions terminated after $20 \mathrm{~min}$ passed without reward delivery. Following stable responding on the PR task (typically, four to eight sessions), FSCV recordings were performed every other session.

Sucrose consumption and open-field locomotion. JZL effects on overall motor function and sucrose consumption were assessed on 2 consecutive days following treatment with JZL ( $8 \mathrm{mg} / \mathrm{kg}$, i.p.) or VEH $120 \mathrm{~min}$ before session onset. Sucrose pellets were placed in a plastic cup in the center of a $1 \times 1 \mathrm{~m}$ arena and locomotion was monitored for $30 \mathrm{~min}$ using a digital video camera, positioned overhead. Locomotion was analyzed using EthoVision XT (Noldus) and sucrose consumption was calculated as the weight consumed during this period.

Pharmacology. Mice in the drug treatment group were randomly assigned to receive a low dose $(8 \mathrm{mg} / \mathrm{kg}$, i.p.) of the MAGL inhibitor JZL or VEH for six consecutive sessions, and then the opposite treatment for six consecutive sessions. VEH and JZL treatment occurred $120 \mathrm{~min}$ before session onset (Long et al., 2009). JZL was dissolved in the 1:1:18 VEH consisting of Emulphor, ethanol, and saline, respectively.

Experimental design and statistical analysis. Behavior and voltammetric measures were analyzed using a one- or two-way repeated-measures (RM) ANOVA, or unpaired $t$ tests. Significance was set at $p<0.05$ and Holm-Sidak post hoc test was used to correct for multiple comparisons when appropriate. Statistical analyses were performed in Prism (version 6; GraphPad). Statistical details for each experiment are presented in figure legends. The $n$ for each experiment is presented in the figure legends or in Materials and Methods.

\section{Results}

\section{In vivo recording of $\mathrm{DA}$ release during a $\mathrm{PR}$ chain schedule of reinforcement}

A carbon fiber microelectrode was implanted in the NAc to probe subsecond DA concentration changes using FSCV, and mice were trained to respond on a modified $\mathrm{PR}$, chain schedule 


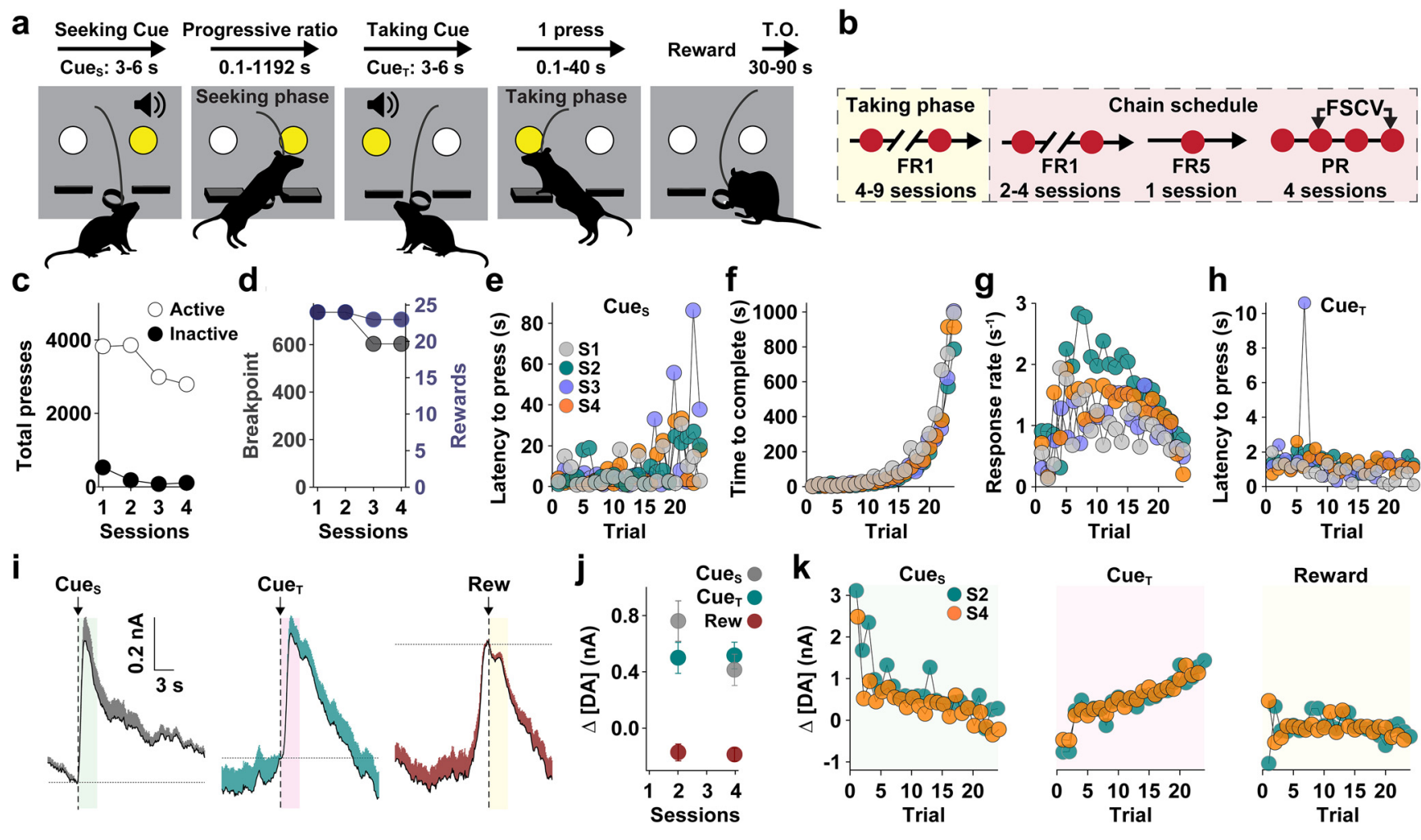

Figure 1. Experimental design and representative data. $\boldsymbol{a}$, Schematic of behavioral sequence on each trial of the chain schedule. Values beneath each event represent the range across all animals $(n=32)$. $\boldsymbol{b}$, Behavioral training and FSCV recording schedule. $\boldsymbol{c}-\boldsymbol{k}$, Representative metrics from one mouse. $\boldsymbol{c}$, Total presses on the Active and Inactive levers. $\boldsymbol{d}$, Final break point and total number of rewards earned across consecutive sessions. $\boldsymbol{e}-\boldsymbol{h}$, Trial-by-trial changes on consecutive sessions (S1-S4) in latency to initiate pressing during the seeking phase $(\boldsymbol{e})$, time to complete response requirement $(\boldsymbol{f})$, response rate during the seeking phase $(\boldsymbol{g})$, and latency to press during the\& "taking phase" $(\boldsymbol{h}) . \boldsymbol{i}$, Mean $( \pm$ SEM) dopamine concentration change $(\Delta[D A])$ relative to stimulus onset (vertical line). Horizontal line represents the reference point (i.e., $0 \mathrm{nA}$ ) for quantifying $\Delta[D A]$. $\boldsymbol{j}$, Mean ( \pm SEM) peak $\Delta[D A]$ across all sessions. $\boldsymbol{k}$, Trial-by-trial peak $\Delta[D A]$ relative to each stimulus.

of sucrose reinforcement. The behavioral task, training, and recording schedule, and representative data from one mouse across consecutive PR sessions is shown in Figure 1. The instrumental sequence on each trial is shown in Figure $1 a$. Mice first responded on one of two levers (the $\mathrm{Lev}_{\mathrm{S}}$ ) following presentation of the $\mathrm{Cue}_{\mathrm{S}}$, which occurred after a variable timeout period so that the opportunity to work for reward was unpredictable and salient. A random duration for $\mathrm{Cue}_{\mathrm{S}}$ (range, 3-6 s) was used to mitigate the effects of timing on DAergic encoding of reward expectation (Oleson et al., 2014; Soares et al., 2016; Howard et al., 2017). To assess motivation, the response requirement exponentially increased on each trial according to a PR schedule (Hodos, 1961; Covey et al., 2016). Upon reaching the response requirement, both levers retracted and the $\mathrm{Cue}_{\mathrm{T}}$ (range, 3-6 s) signaled that a single response on the opposite $\left(\mathrm{Lev}_{\mathrm{T}}\right)$ would earn one sucrose pellet. Thus, the effortful and temporal cost of reward increased on each trial, while the moment of reward delivery remained predictable. This approach allowed us to differentiate how DA neurons respond to cues signaling increasing cost of reward versus the reward itself.

Figure 1 displays measures from a representative mouse across consecutive sessions (Fig. 1c,d,i,j) and consecutive trials within each session (Fig. 1e-h,k). As reinforcer cost increased across the PR session, the on each trial (Fig. 1e) and time to complete each trial (Fig. 1f) increased, while response rate (lever presses per second) initially increased and then declined during high cost trials (Fig. 1g). Latency to press $\operatorname{Lev}_{\mathrm{T}}$, which remained at an FR1 requirement on each trial, did not change across the session (Fig. 1h). DA release, averaged across the entire session, signaled the opportunity to work for reward $\left(\mathrm{Cue}_{\mathrm{S}}\right)$ and experienced $\left(\mathrm{Cue}_{\mathrm{T}}\right)$ cost of reward, respectively, but did not change in response to eventual reward (Rew) delivery (Fig. $1 i, j$ ). Trial-by-trial measures show that cue-evoked DA release changed in a diametric fashion as reward cost increased (Fig. $1 k$ ), decreasing or increasing at trial onset or offset, respectively, as a function of escalating reward costs.

Figure 2 highlights that DA release-averaged across the entire session for all animals-is similar for Cue $\mathrm{S}_{\mathrm{S}}$ and $\mathrm{Cue}_{\mathrm{T}}$ (Fig. $2 a, b)$, but these signals change in the opposite manner over time, when comparing the first versus the second half of the session (Fig. 2c,d). Gross behavioral measures (break point, reward count, and total active lever presses) did not vary according to session (two-way RM ANOVA; all $p>0.89$ ) or sex (two-way RM ANOVA; all $p>0.16$ ). We therefore collapsed values across sessions and sex for subsequent analyses.

To more specifically assess DAergic encoding of motivation, we focused on later trials when cost is greatest by analyzing the final 10 trials of the PR session for each animal, normalized according to individual break points (Fig. 3; Wanat, 2010; Covey et al., 2016, 2018). Latency to initiate responding increased on each trial (Fig. 3a) as cost increased and the response rate decreased (Fig. $3 b$ ), both contributing to a greater time to complete the response requirement on each trial (Fig. $3 c$ ). In contrast, the latency to take reward, once available, did not change (Fig. $3 a$ ). Stimulus-entrained DA release also differentially tracked reinforcer cost. DA release following $\mathrm{Cue}_{S}$, which signaled a greater future cost on each subsequent trial, declined, while DA release to $\mathrm{Cue}_{\mathrm{T}}$, which signaled completion of subsequently greater response requirements, increased, and DA release to 
a
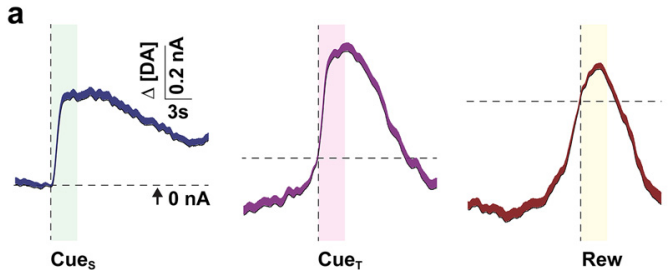

C

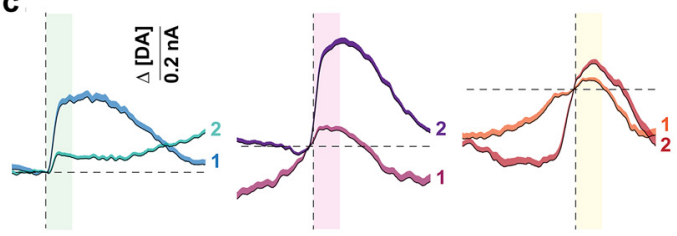

b

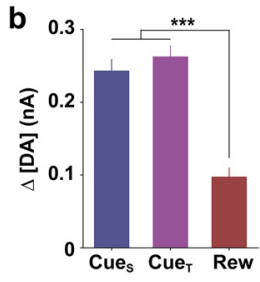

d

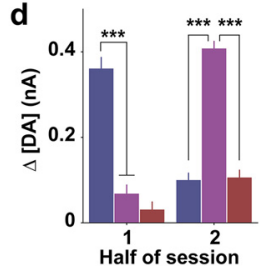

Figure 2. Stimulus- and time-specific changes in dopamine release. $\boldsymbol{a}$, Mean ( \pm SEM) DA concentration change $(\Delta[D A])$ relative to stimulus onset (vertical line) across all mice and sessions. Horizontal line represents the reference point (i.e., $0 \mathrm{nA})$ for quantifying $\Delta[D A]$. $\boldsymbol{b}-\boldsymbol{d}$, Mean ( \pm SEM) peak $\Delta[\mathrm{DA}]$ across all sessions (one-way ANOVA; $F_{(2,1719)}=33.03$, ${ }^{* * *} p<0.001 ; \boldsymbol{b}$ ) and separated into bins representing one-half of the total trials $(\boldsymbol{c}, \boldsymbol{d})$, relative to each stimulus (two-way RM ANOVA; time $\times$ stimulus, $\left.F_{(4,570)}=70.44\right)$. Post hoc comparison of $\Delta[D A]$ relative to each stimulus, ${ }^{* * *} p<0.001$.
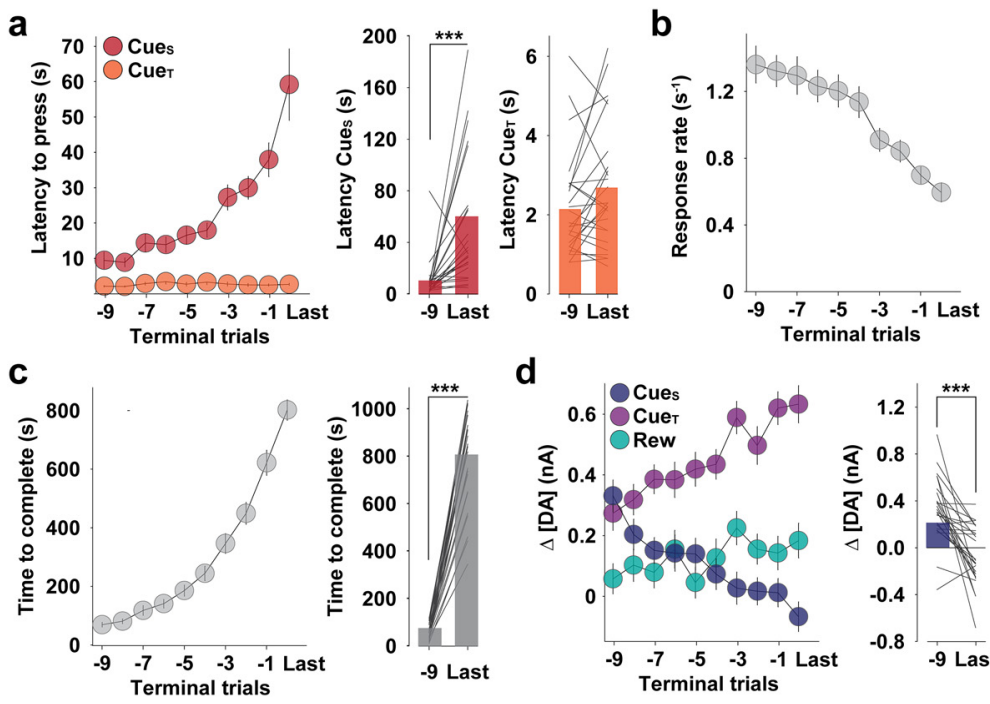

Figure 3. Diametric encoding of reward cost. In all panels, the left panel displays overall mean ( \pm SEM) across the final 10 trials; the right panel displays individual values on trial " -9 " and "last" trial (bars display overall mean and lines display value for each mouse). $\boldsymbol{a}-\boldsymbol{d}$, Latency to initiate pressing following trial onset (Cues) and after reaching response requirement (Cue ; two-way RM ANOVA; trial $\times$ cue: $F_{(9,450)}=10.77, p<0.001 ; \boldsymbol{a}$ ), response rate (one-way RM ANOVA: $F_{(9,225)}=$ $17.45, p<0.001 ; \boldsymbol{b})$, time to complete response requirement (one-way RM ANOVA: $F_{(9,225)}=121.80, p<0.001$; $\boldsymbol{c}$, right), and $\Delta[D A]$ relative to stimulus onset (two-way RM ANOVA: trial $\times$ stimulus: $F_{(18,67)}=8.58, p<0.001 ; \boldsymbol{d}$ ) varied according to cost threshold. Post hoc paired $t$ test; ${ }^{* * *} p<0.001,-9$ versus last trial.

eventual reward delivery did not change (Fig. $2 d$ ). Across terminal trials of each session, there was a strong correlation between the response ratio and DA release to $\mathrm{Cue}_{\mathrm{S}}(r=-0.79, p<0.001)$ and $\mathrm{Cue}_{\mathrm{T}}(r=0.93, p<0.001)$. There was also a negative correlation between the latency to initiate reward seeking by pressing $\mathrm{Lev}_{\mathrm{S}}$ and DA release to $\mathrm{Cue}_{\mathrm{S}}(r=-0.76, p<0.01)$ and $\mathrm{Cue}_{\mathrm{T}}$ $(r=0.89, p<0.001)$, while the latency to press $\operatorname{Lev}_{\mathrm{T}}$ weakly correlated with DA release to $\mathrm{Cue}_{\mathrm{S}}(r=-0.28, p=0.40)$ and $\mathrm{Cue}_{\mathrm{T}}(r=$ $-0.15, p=0.70)$. Collectively, as cost increases on each trial, the propensity to initiate reward seeking and DA release at trial onset declined, while release increased following completion of greater effortful investments, but reward cost did not influence DA release to the reward itself.
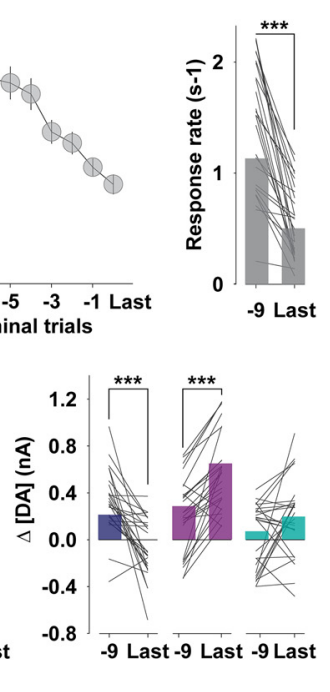

Repeated inhibition of 2-AG

degradation consistently increases motivation and DA function

Our prior work demonstrates that increasing tissue levels of 2-AG following acute treatment with a high dose of the MAGL inhibitor JZL ( $18-40 \mathrm{mg} / \mathrm{kg}$, i.p.) augments motivation to work for reward (Covey et al., 2018; Oleson et al., 2012). Because repeated treatment with highdose JZL $(16 \mathrm{mg} / \mathrm{kg}$, i.p.) produces rapid CB1 receptor desensitization and behavioral tolerance (Baggelaar et al., 2018), it is critical to determine whether a lower dose of JZL remains effective over time. To assess whether repeated inhibition of 2-AG degradation supports prolonged increases in motivation, we administered low-dose JZL (8 mg/kg, i.p.) for six consecutive sessions (Fig. 4a). JZL significantly and consistently increased reward seeking (Fig. 4b-d). To determine whether prior treatment with JZL influenced future behavior, we compared the final break point on the first day of drug treatment (i.e., sessions 1 and 7) and found a significant effect of drug (twoway RM ANOVA: drug: $F_{(1,34)}=9.90$, $p<0.01)$, but no effect of session $(p=0.182)$ or interaction $(p=0.552)$, indicating that treatment order did not influence drug effect. Gross behavioral measures (break point, reward count, and total active lever presses) also did not vary according to sex (sex: all $p>0.16$; sex $\times$ drug, all $p>0.28$ ). We therefore collapsed values across sessions and sex for subsequent analyses. Moreover, we assessed whether JZL indiscriminately increased lever pressing by comparing $\operatorname{Lev}_{\mathrm{T}}$ presses during the seeking phase. The number of $\mathrm{Lev}_{\mathrm{T}}$ presses per trial during the seeking phase (total $\mathrm{Lev}_{\mathrm{T}}$ presses divided by total trial number to correct for increased trial count following JZL treatment) remained low following both VEH $(2.40 \pm 0.22)$ and JZL $(2.88 \pm 0.23)$ treatment, and no difference was observed between treatment groups $\left(t_{(36)}=1.33, p=0.19\right)$.

Figure 5 highlights that DA release, averaged across all animals, exhibited a similar pattern in VEH and JZL treatment groups across the entire session (Fig. 5a,b). A similar pattern to that described above (Fig. 2) was observed when comparing the first versus the second half of the session (Fig. $5 c, d$ ). Specifically, DA release preferentially responds to predictive cues versus reward delivery, but does so in an opposite manner, decreasing in response to $\mathrm{Cue}_{\mathrm{S}}$ while increasing in response to $\mathrm{Cue}_{\mathrm{T}}$. Moreover, JZL treatment selectively augments DA release to $\mathrm{Cue}_{\mathrm{T}}$. To further assess the behavioral actions contributing to increased PR performance and their DAergic correlates, we analyzed behavior and DA measures across the final trials of each PR session, as mice reached their respective break points (Fig. 6). JZL increased the 
propensity to initiate reward seeking following presentation of $\mathrm{Cue}_{\mathrm{S}}$ (Fig. 6a,b) and attenuated the decline in response rate (Fig. $6 c, d$ ), while the latency to access reward, once available, did not change (Fig. 6e,f). Although JZL decreased the latency to initiate reward seeking on each trial, DA release at trial onset (i.e., $\mathrm{Cue}_{\mathrm{S}}$ ) was not altered (Fig. $6 g, h$ ). Yet, given that the response requirement was greater during terminal trials following JZL treatment, DA release per future unit cost was greater following JZL treatment. In contrast, JZL increased DA release to $\mathrm{Cue}_{\mathrm{T}}$ as a function of the prior response requirement (Fig. 6i,j), while DA release to reward delivery remained low in both groups (Fig. $6 k, l)$.

Finally, to determine whether JZL facilitated effortful responding by increasing appetite or acting as a psychostimulant, we monitored "free-access" sucrose consumption and open-field locomotion (Fig. 7). JZL treatment did not alter sucrose intake (Fig. 7a), distance traveled (Fig. $7 b$ ), or movement velocity (Fig. $7 c$ ), indicating a selective invigoration of goaldirected action.

\section{Discussion}

Apathy is characterized by diminished willingness to overcome effortful costs, independent of changes in motor function, cognitive impairment, or mood (Stahl, 2002; Levy and Dubois, 2006; Friedman et al., 2007; Salamone et al., 2017). Motivational deficits are generally treated with antidepressants such as selective serotonin reuptake inhibitors, but these have been found to be ineffective at improving effort-related aspects of motivation (Cooper et al., 2014) and often worsen symptoms of apathy by diminishing the hedonic impact of rewards (Hoehn-Saric et al., 1990; Yohn et al., 2016). Alternatively, DA transporter inhibitors, such as bupropion, D-amphetamine, or methylphenidate, facilitate motivated behaviors, but possess undesirable motoric side effects and high abuse liability (Salamone et al., 2016). Therefore, pharmacological strategies are required that promote effortful action without altering normal reward processing. An important step in this process is identifying how potential treatments alter individual components of reward-directed action sequences and their neural correlates.

The general consensus is that cue-evoked accumbal DA release and phasic DA neuron firing preferentially signal the expected reward size, delay, and probability (Wanat et al., 2010; Cohen et al., 2012; Pasquereau and Turner, 2013; Hollon et al., 2014; Eshel et al., 2015; Covey and Cheer, 2019), but not the cost (Gan et al., 2010; Wanat et al., 2010; Pasquereau and Turner, 2013; Hollon et al., 2014). However, other reports find that accumbal DA release scales directly according to effortful cost $p=0.296 ; \boldsymbol{d})$

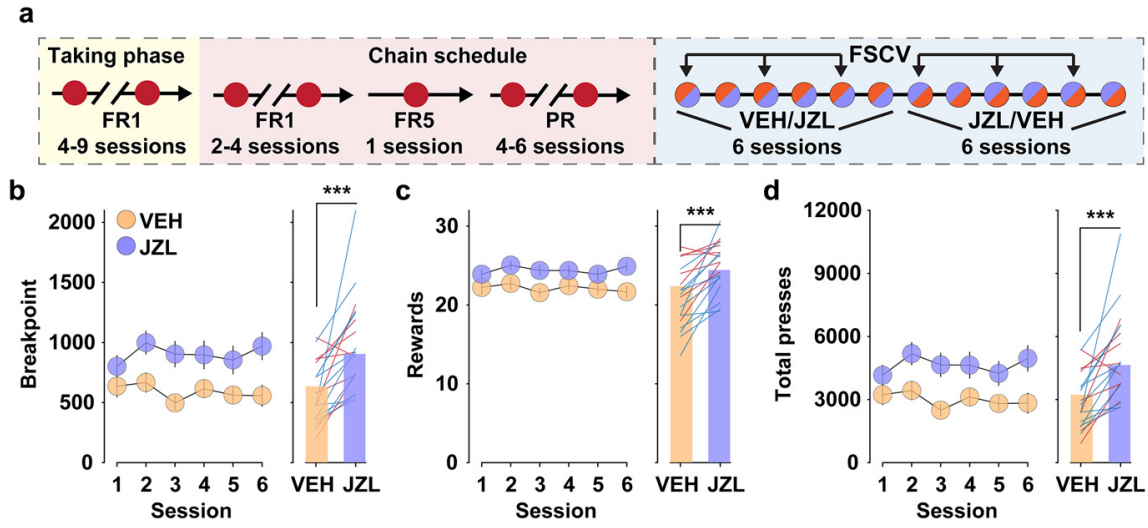

Figure 4. Chronic augmentation of eCB signaling increases motivation. $\boldsymbol{a}$, Experimental design. $\boldsymbol{b}-\boldsymbol{d}$, Left, Mean ( \pm SEM) value on each session, compared using a two-way RM ANOVA. Right, The overall (bars) and individual (lines; blue, female; and total lever presses (drug: $F_{(1,36)}=8.99, p<0.01$; session: $F_{(5,180)}=1.96, p=0.103$; drug $\times$ session: $F_{(5,180)}=1.23$, a

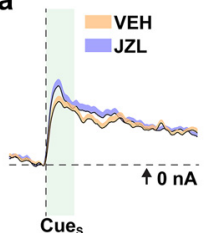

C

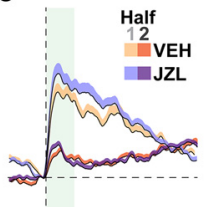

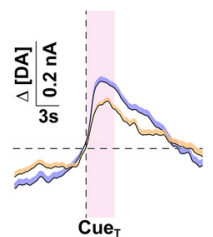

cue $_{T}$

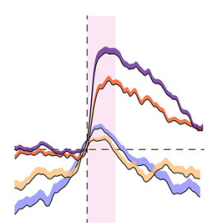

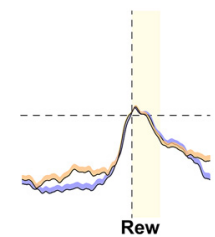

Rew

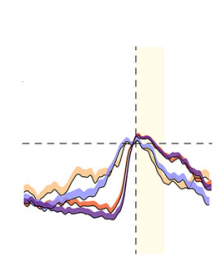

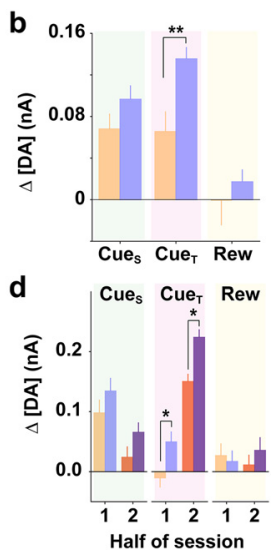

Figure 5. JZL alters stimulus-specific changes in dopamine release. $\boldsymbol{a}$, Mean (+SEM) DA concentration change $(\Delta[D A])$ relative to stimulus onset (vertical line) across all mice and sessions following VEH or JZL treatment. Horizontal line represents the reference point (i.e., $0 \mathrm{nA}$ ) for quantifying $\triangle[D A]$. $\boldsymbol{b}-\boldsymbol{d}$, Mean ( $\pm S E M$ ) stimulus-evoked across all sessions and treatments (two-way ANOVA; stimulus: $F_{(2,2514)}=15.20, p<0.001$; drug: $F_{(1,2514}$ $=7.98, p<0.01 ; \boldsymbol{b})$ and separated into bins representing one-half of the total trials, relative to each stimulus $(\boldsymbol{c}, \boldsymbol{d})$. Separate two-way RM ANOVAs revealed a significant effect of time for $\Delta[D A] C_{e_{S}}\left(F_{(1,419)}=12.12\right.$,

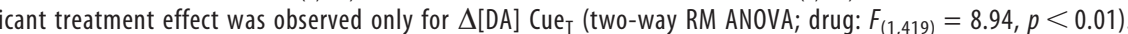
Post hoc comparison of $\Delta[\mathrm{DA}]$ relative to $\mathrm{VEH},{ }^{*} p<0.05,{ }^{* *} p<0.01$.

(Schelp et al., 2017). Here, we provide further support that accumbal DA release signals reward cost. We used a chain schedule of reinforcement and PR response requirement to decipher how behavior and DA function change in response to distinct stimuli that signal future and prior reward cost versus reward receipt. We observed diametric DAergic encoding of cues signaling effort initiation and completion in the NAc (Fig. 2). DA release at trial onset $\left(\mathrm{Cue}_{\mathrm{S}}\right)$ declines as cost increases, potentially dissuading the subject to work for reward (Eban-Rothschild et al., 2016), as reflected by an increased latency to initiate responding and decreased response rate across the trial. Diminished DA release may reflect the exponential decrease in expected reward value (price per unit) on each trial. If decreasing DA release to $\mathrm{Cue}_{\mathrm{S}}$ reflects discounted reward value because of increasing future cost, then JZL treatment may diminish perceived cost and 

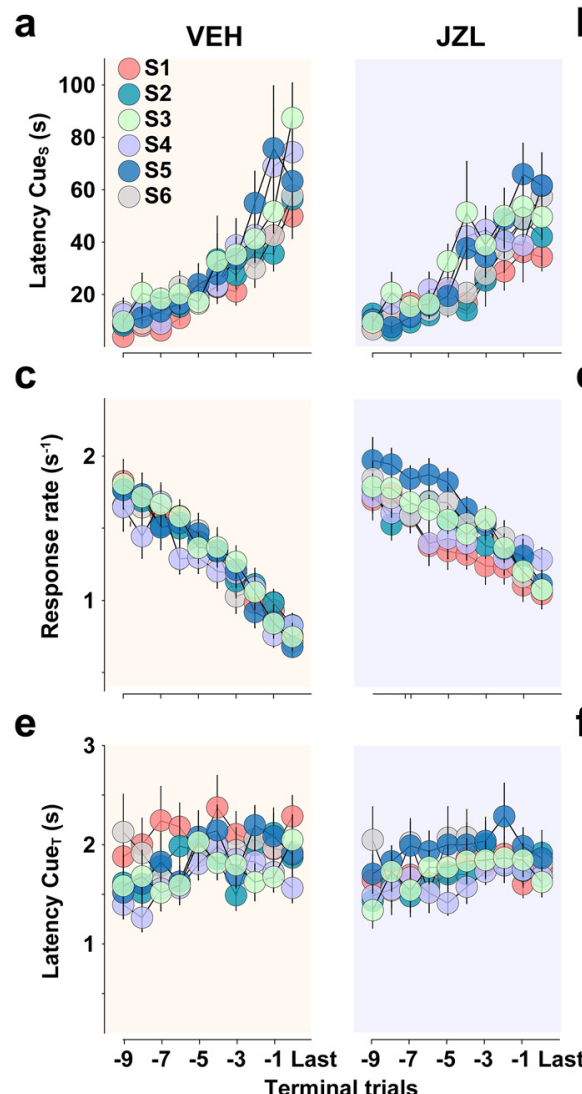

b

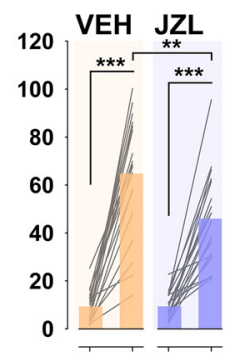

d

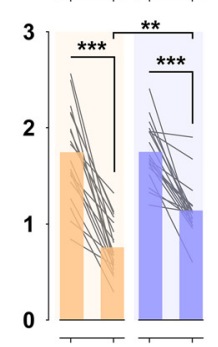

f

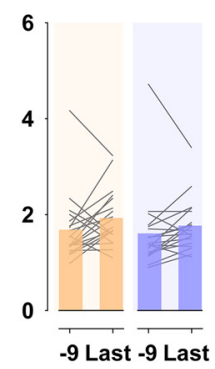

g

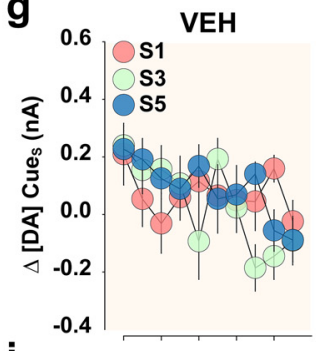

i
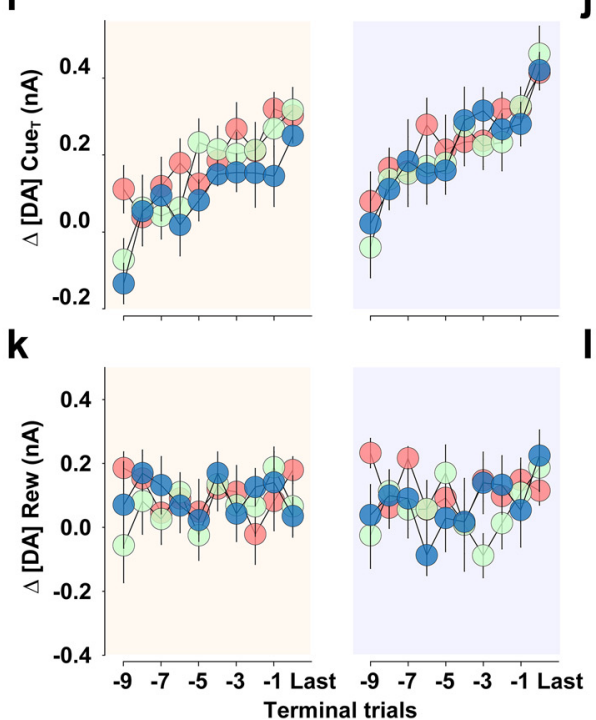

h
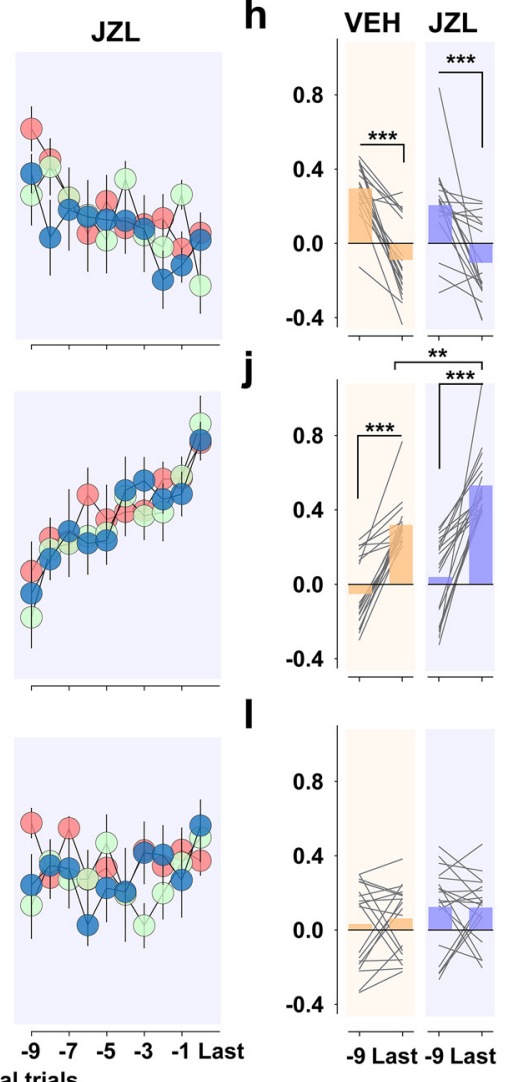

Figure 6. Chronic augmentation of eCB signaling invigorates reward seeking and dopamine function. In all panels, the left panel displays the mean ( \pm SEM) across terminal trials on each of sessions 1-6 (S1-S6); the right panel displays the overall (bars) and individual (lines) mean, collapsed across sessions. Datasets in the left and right panels were compared using a two-way RM ANOVA. $\boldsymbol{a}, \boldsymbol{b}$, Latency to press after trial onset (Cues) increased across trials (VEH: trial: $F_{(9,162)}=29.04, p<0.001$; session: $F_{(5,90)}=2.07, p=0.077$; trial $\times$ session: $F_{(45,972)}=1.09$, $p=0.328$; JZL: trial: $F_{(9,162)}=25.20, p<0.001$; session: $F_{(5,90)}=1.90, p=0.102$; trial $\times$ session: $\left.F_{(45,972)}=1.23, p=0.149 ; \boldsymbol{a}\right)$ and JZL attenuated this increase (trial $\times$ treatment:\& $F_{(1,36)}=$ $6.96, p=0.012 ; \boldsymbol{b})$. $\boldsymbol{c}, \boldsymbol{d}$, Response rate decreased across trials (VEH, trial, $F_{(9,162)}=57.01, p<0.001$; session, $\mathrm{F}_{(5,90)}=0.45, p=0.813$; trial $\times$ session, $F_{(45,972)}=0.71, p=0.922 ;$ JZL, trial, $F_{(9,162)}=40.29, p<0.001 ;$ session, $F_{(5,90)}=1.96, p=0.092 ;$ trial $\times$ session: $\left.F_{(45,972)}=1.47, p=0.026 ; \boldsymbol{c}\right)$ and JZL attenuated this decrease (trial $\times$ treatment: $\left.F_{(1,36)}=4.97, p=0.032 ; \boldsymbol{d}\right)$. $\boldsymbol{e}, \boldsymbol{f}$, Latency to press on the Cue did not change across trials (VEH: trial: $F_{(9,162)}=1.17, p=0.318$; session: $F_{(5,90)}=1.74, p=0.167$; trial $\times$ session: $F_{(45,972)}=0.99, p=0.452$; JZL: trial: $F_{(9,162)}=2.27, p=0.064$; session: $F_{(5,90)}=2.40, p=0.063 ;$ trial $\times$ session: $\left.F_{(45,972)}=1.05, p=0.407 ; \boldsymbol{e}\right)$ and was unaffected by JZL (trial $\times$ treatment: $F_{(1,36)}=$ $0.002, p=0.964 ; \boldsymbol{f}) . \boldsymbol{g}, \boldsymbol{h}, \Delta[\mathrm{DA}] \mathrm{Cue}_{\mathrm{s}}$ decreased across trials (VEH: trial: $F_{(9,162)}=3.09, p<0.01$; session: $F_{(2,54)}=0.52, p=0.605 ;$ trial $\times$ session: $F_{(18,486)}=1.49$, $p=0.099$; JZL: trial: $F_{(9,162)}=3.07, p<0.01$; session: $F_{(2,54)}=0.95, p=0.408$; trial $\times$ session: $\left.F_{(18,486)}=0.97, p=0.503 ; \boldsymbol{g}\right)$ but was unaffected by JZL (trial: $F_{(1,36)}=$ 63.11, $p<0.001$; treatment: $F_{(1,36)}=1.16, p=0.288$; trial $\times$ treatment: $\left.F_{(1,36)}=0.71, p=0.407 ; \boldsymbol{h}\right) . \boldsymbol{i}, \boldsymbol{j}, \Delta[\mathrm{DA}]$ Cue $\mathrm{T}_{\mathrm{T}}$ increased across trials (VEH: trial: $F_{(9,162)}=11.89$, $p<0.001$; session: $F_{(2,54)}=0.13, p=0.880$; trial $\times$ session: $F_{(18,486)}=0.50, p=0.956$; JZL: trial: $F_{(9,162)}=9.74, p<0.001$; session: $F_{(2,54)}=1.24, p=0.306 ;$ trial $\times$ session: $\left.F_{(18,486)}=1.08, p=0.378 ; i\right)$, and JZL augmented this increase (trial: $F_{(1,36)}=119.50, p<0.001$; treatment: $F_{(1,36)}=12.50, p<0.01$; trial $\times$ treatment: $F_{(1,36)}=$ $2.37, p=0.133 ; j) . \boldsymbol{k}, I, \Delta[\mathrm{DA}]_{\text {Rew }}$ did not change across trials (VEH: trial: $F_{(9,162)}=0.79, p<0.585$; session: $F_{(2,54)}=0.26, p=0.775 ;$ trial $\times$ session: $F_{(18,486)}=0.58$, $p=0.912$; JZL: trial: $F_{(9,162)}=1.24, p=0.292$; session: $F_{(2,54)}=0.82, p=0.451$; trial $\times$ session: $\left.F_{(18,486)}=1.02, p=0.362 ; \boldsymbol{k}\right)$ and was unaffected by JZL (trial: $F_{(1,36)}=$ $0.13, p=0.662$; treatment: $F_{(1,36)}=1.93, p=0.173$; trial $\times$ treatment: $\left.F_{(1,36)}=0.19, p=0.662 ; l\right)$. Post hoc $t$ tests: ${ }^{* * *} p<0.001,{ }^{* *} p<0.01$.
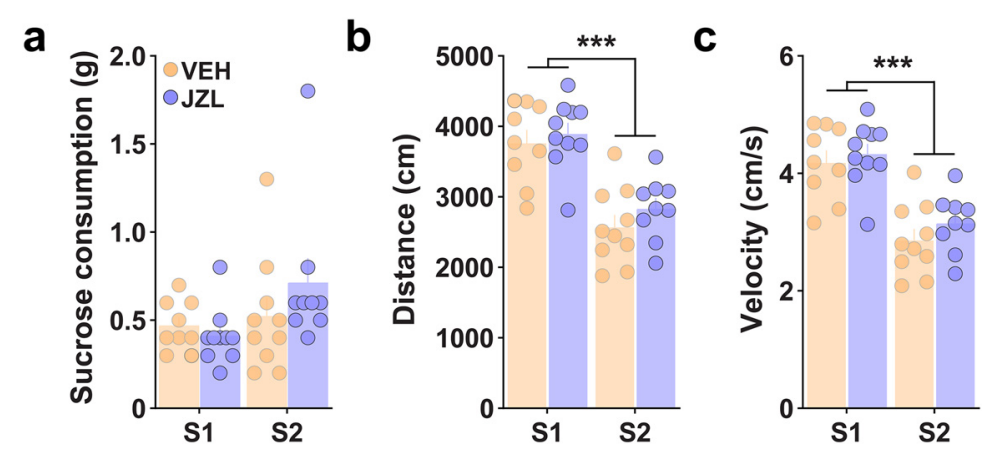

Figure 7. MAGL inhibition does not alter consumption or locomotion. $\boldsymbol{a}-\boldsymbol{c}, \mathrm{JZL}(8 \mathrm{mg} / \mathrm{kg}$, i.p.) treatment across two consecutive sessions $(S 1, S 2)$ did not alter free-access sucrose consumption (two-way RM ANOVA; treatment: $F_{(1,34)}=0.52$, $p=0.475 ; \boldsymbol{a})$ or open-field locomotion measured by distance traveled (two-way RM ANOVA; treatment: $F_{(1,34)}=1.42$, $p=0.249 ; \boldsymbol{b}$ ) and movement velocity (two-way RM ANOVA; treatment: $F_{(1,34)}=1.42, p=0.249 ; \boldsymbol{c}$ ). Post hoc comparison following significant main effect of time, S1 vs $52,{ }^{* *} p<0.001$. facilitate task engagement (i.e., latency to initiate pressing) by maintaining DA release to $\mathrm{Cue}_{\mathrm{S}}$ at greater relative levels per future cost. However, additional work is clearly required to understand how DA release precisely influences cost perception.

In contrast, the gradual increase in DA release evoked by trial completion $\left(\mathrm{Cue}_{\mathrm{T}}\right)$ may reflect a positive prediction error. While reward value declines according to the increased cost and delay (McClure et al., 2004), cues that resolve uncertainty about future reward availability can serve as powerful conditioned reinforcers that drive information seeking (Bromberg-Martin and Hikosaka, 
2009). As the delay from trial onset increases on each trial (Fig. 2c), if and when reward will become available becomes more uncertain. Accordingly, $\mathrm{Cue}_{\mathrm{S}}$ becomes a worse predictor of reward availability, while $\mathrm{Cue}_{\mathrm{T}}$ becomes more informative. The degree to which increasing DA release reflects salience or uncertainty that arises from either a greater delay to reinforcement versus the amount of exerted effort is unclear, as these metrics are highly correlated. However, the behavioral task structure allowed us to determine that increased DA release at trial completion, observed here and in prior work (Wanat et al., 2010, 2013; Covey et al., 2016, 2018 ), is not because of DA neurons assigning greater value to a reward that is earned following greater work requirements, as previously suggested (Wanat et al., 2010). Rather, accumbal DA release preferentially responds to cues that signal increasing future and prior cost of a reward versus a reward receipt itself.

The eCB system serves a prominent role in controlling motivated behavior and DA neurotransmission, and may represent a viable target for disorders of motivation. Our prior work demonstrates that increasing tissue levels of 2AG with JZL facilitates the motivation to work for reward and NAc DA release (Covey et al., 2018). MAGL inhibitors such as JZL likely represent a superior therapeutic strategy compared with direct CB1 receptor agonists such as $\delta-9$ tetrahydrocannabinol (Grabner et al., 2017; Mulvihill and Nomura, 2013), as direct $\mathrm{CB} 1$ receptor agonists reduce effortful behaviors (Fatahi et al., 2018; Fatahi and Haghparast, 2018). In contrast to agonists that indiscriminately target $\mathrm{CB} 1$ receptors throughout the brain, MAGL inhibitors indirectly increase $\mathrm{CB} 1$ receptor binding by amplifying ongoing 2-AG signaling. Within the VTA, 2-AG mobilization suppresses inhibitory inputs onto DA neurons via activation of presynaptic $\mathrm{CB} 1$ receptors, increasing cell firing and downstream accumbal DA release (Wang and Lupica, 2014). However, the therapeutic potential of JZL for disorders of motivation requires that repeated augmentation of this circuit consistently facilitates effortful responding and DA function. Notably, repeated treatment with high-dose JZL $(40 \mathrm{mg} / \mathrm{kg})$ produces rapid tolerance to its behavioral actions and downregulation of $\mathrm{CB} 1$ receptors (Schlosburg et al., 2010). Here, we used a lower dose of JZL $(8 \mathrm{mg} / \mathrm{kg})$ and observed a stable, nondesensitizing increase in behavioral output and NAc DA release across all sessions. Our findings corroborate prior work demonstrating that chronic, low-dose JZL $(\leq 8 \mathrm{mg} / \mathrm{kg}$ ) does not lead to tolerance under other experimental conditions (Sumislawski et al., 2011; Ghosh et al., 2013). Importantly, JZL did not function as a locomotor stimulant and failed to alter sucrose consumption when feely available (Fig. 7). Additionally, JZL does not exhibit rewarding or aversive properties in place conditioning assays (Gamage et al., 2015; Muldoon et al., 2015). Therefore, cannabinoid receptor-based therapies such as MAGL inhibition may represent a viable treatment for motivational dysfunctions that are a hallmark of numerous psychiatric and neurologic disorders.

\section{References}

Baggelaar MP, Maccarrone M, van der Stelt M (2018) 2-Arachidonoylglycerol: a signaling lipid with manifold actions in the brain. Prog Lipid Res 71:1-17.

Bromberg-Martin ES, Hikosaka O (2009) Midbrain dopamine neurons signal preference for advance information about upcoming rewards. Neuron 63:119-126.
Cheer JF, Wassum KM, Sombers LA, Heien ML, Ariansen JL, Aragona BJ, Phillips PE, Wightman RM (2007) Phasic dopamine release evoked by abused substances requires cannabinoid receptor activation. J Neurosci 27:791-795

Cohen JY, Haesler S, Vong L, Lowell BB, Uchida N (2012) Neuron-type-specific signals for reward and punishment in the ventral tegmental area. Nature 482:85-88.

Cooper JA, Tucker VL, Papakostas GI (2014) Resolution of sleepiness and fatigue: a comparison of bupropion and selective serotonin reuptake inhibitors in subjects with major depressive disorder achieving remission at doses approved in the European Union. J Psychopharmacol 28:118-124.

Covey DP, Cheer JF (2019) Accumbal dopamine release tracks the expectation of dopamine neuron-mediated reinforcement. Cell Rep 27:481490.e3.

Covey DP, Dantrassy HM, Yohn SE, Castro A, Conn PJ, Mateo Y, Cheer JF (2018) Inhibition of endocannabinoid degradation rectifies motivational and dopaminergic deficits in the Q175 mouse model of Huntington's disease. Neuropsychopharmacology 43:2056-2063.

Covey DP, Dantrassy HM, Zlebnik NE, Gildish I, Cheer JF (2016) Compromised dopaminergic encoding of reward accompanying suppressed willingness to overcome high effort costs is a prominent prodromal characteristic of the Q175 mouse model of Huntington's disease. J Neurosci 36:4993-5002.

Eban-Rothschild A, Rothschild G, Giardino WJ, Jones JR, de Lecea L (2016) VTA dopaminergic neurons regulate ethologically relevant sleep-wake behaviors. Nat Neurosci 19:1356-1366.

Eshel N, Bukwich M, Rao V, Hemmelder V, Tian J, Uchida N (2015) Arithmetic and local circuitry underlying dopamine prediction errors. Nature 525:243-246.

Fatahi Z, Haghparast A (2018) Activation of the cannabinoid system in the nucleus accumbens affects effort-based decision making. Pharmacol Biochem Behav 165:29-35.

Fatahi Z, Reisi Z, Rainer G, Haghparast A, Khani A (2018) Cannabinoids induce apathetic and impulsive patterns of choice through $\mathrm{CB} 1$ receptors and TRPV1 channels. Neuropharmacology 133:75-84.

Friedman JH, Brown RG, Comella C, Garber CE, Krupp LB, Lou J-S, Marsh L, Nail L, Shulman L, Taylor CB (2007) Fatigue in Parkinson's disease: a review. Mov Disord 22:297-308.

Gamage TF, Ignatowska-Jankowska BM, Muldoon PP, Cravatt BF, Damaj MI, Lichtman AH (2015) Differential effects of endocannabinoid catabolic inhibitors on morphine withdrawal in mice. Drug Alcohol Depend $146: 7-16$

Gan JO, Walton ME, Phillips PE (2010) Dissociable cost and benefit encoding of future rewards by mesolimbic dopamine. Nat Neurosci 13:25-27.

Ghosh S, Wise LE, Chen Y, Gujjar R, Mahadevan A, Cravatt BF, Lichtman AH (2013) The monoacylglycerol lipase inhibitor JZL184 suppresses inflammatory pain in the mouse carrageenan model. Life Sci 92:498505.

Grabner GF, Zimmermann R, Schicho R, Taschler U (2017) Monoglyceride lipase as a drug target: At the crossroads of arachidonic acid metabolism and endocannabinoid signaling. Pharmacol Ther 175:35-46.

Heien MLAV, Khan AS, Ariansen JL, Cheer JF, Phillips PEM, Wassum KM, Wightman RM (2005) Real-time measurement of dopamine fluctuations after cocaine in the brain of behaving rats. Proc Natl Acad Sci U S A 102:10023-10028.

Hoehn-Saric R, Lipsey JR, McLeod DR (1990) Apathy and indifference in patients on fluvoxamine and fluoxetine. J Clin Psychopharmacol 10:343345.

Hodos W (1961) Progressive ratio as a measure of reward strength. Science 134:943-944.

Hollon NG, Arnold MM, Gan JO, Walton ME, Phillips PEM (2014) Dopamine-associated cached values are not sufficient as the basis for action selection. Proc Natl Acad Sci U S A 111:18357-18362.

Howard CD, Li H, Geddes CE, Jin X (2017) Dynamic nigrostriatal dopamine biases action selection. Neuron 93:1436-1450.

Le Heron C, Holroyd CB, Salamone J, Husain M (2019) Brain mechanisms underlying apathy. J Neurol Neurosurg Psychiatry 90:302-312.

Levy R, Dubois B (2006) Apathy and the functional anatomy of the prefrontal cortex-basal ganglia circuits. Cereb Cortex 16:916-928. 
Long JZ, Nomura DK, Cravatt BF (2009) Characterization of monoacylglycerol lipase inhibition reveals differences in central and peripheral endocannabinoid metabolism. Chem Biol 16:744-753.

McClure SM, Laibson DI, Loewenstein G, Cohen JD (2004) Separate neural systems value immediate and delayed monetary rewards. Science 306:503-507.

Muldoon PP, Chen J, Harenza JL, Abdullah RA, Sim-Selley LJ, Cravatt BF, Miles MF, Chen X, Lichtman AH, Damaj MI (2015) Inhibition of monoacylglycerol lipase reduces nicotine withdrawal. Br J Pharmacol 172:869882 .

Mulvihill MM, Nomura DK (2013) Therapeutic potential of monoacylglycerol lipase inhibitors. Life Sci 92:492-497.

Oleson EB, Beckert MV, Morra JT, Lansink CS, Cachope R, Abdullah RA, Loriaux AL, Schetters D, Pattij T, Roitman MF, Lichtman AH, Cheer JF (2012) Endocannabinoids shape accumbal encoding of cue-motivated behavior via $\mathrm{CB} 1$ receptor activation in the ventral tegmentum. Neuron 73:360-373.

Oleson EB, Cachope R, Fitoussi A, Tsutsui K, Wu S, Gallegos JA, Cheer JF (2014) Cannabinoid receptor activation shifts temporally engendered patterns of dopamine release. Neuropsychopharmacology 39:1441-1452.

Pasquereau B, Turner RS (2013) Limited encoding of effort by dopamine neurons in a cost-benefit trade-off task. J Neurosci 33:8288-8300.

Peters KZ, Oleson EB, Cheer JF (2021) A brain on cannabinoids: the role of dopamine release in reward seeking and addiction. Cold Spring Harb Perspect Med 11:a039305.

Sagheddu C, Muntoni AL, Pistis M, Melis M (2015) Endocannabinoid signaling in motivation, reward, and addiction. Int Rev Neurobiol 125:257302 .

Salamone JD, Correa M (2012) The mysterious motivational functions of mesolimbic dopamine. Neuron 76:470-485.

Salamone JD, Yohn SE, López-Cruz L, San Miguel N, Correa M (2016) Activational and effort-related aspects of motivation: neural mechanisms and implications for psychopathology. Brain 139:13251347.

Salamone JD, Correa M, Yohn SE, Yang J-H, Somerville M, Rotolo RA, Presby RE (2017) Behavioral activation, effort-based choice, and elasticity of demand for motivational stimuli: basic and translational neuroscience approaches. Motiv Sci 3:208-229.

Schelp SA, Pultorak KJ, Rakowski DR, Gomez DM, Krzystyniak G, Das R, Oleson EB (2017) A transient dopamine signal encodes subjective value and causally influences demand in an economic context. Proc Natl Acad Sci U S A 114:E11303-E11312.

Schlosburg JE, Blankman JL, Long JZ, Nomura DK, Pan B, Kinsey SG, Nguyen PT, Ramesh D, Booker L, Burston JJ, Thomas EA, Selley DE, Sim-Selley LJ, Liu Q-s, Lichtman AH, Cravatt BF (2010) Chronic monoacylglycerol lipase blockade causes functional antagonism of the endocannabinoid system. Nat Neurosci 13:11131119

Schultz W (2002) Getting formal with dopamine and reward. Neuron 36:241-263.

Schultz W (2015) Neuronal reward and decision signals: from theories to data. Physiol Rev 95:853-951.

Soares S, Atallah BV, Paton JJ (2016) Midbrain dopamine neurons control judgment of time. Science 354:1273-1277.

Stahl SM (2002) The psychopharmacology of energy and fatigue. J Clin Psychiatry 63:7-8.

Sumislawski JJ, Ramikie TS, Patel S (2011) Reversible gating of endocannabinoid plasticity in the amygdala by chronic stress: a potential role for monoacylglycerol lipase inhibition in the prevention of stress-induced behavioral adaptation. Neuropsychopharmacology 36:2750-2761.

Walton ME, Bouret S (2019) What is the relationship between dopamine and effort? Trends Neurosci 42:79-91.

Wanat MJ, Bonci A, Phillips PE (2013) CRF acts in the midbrain to attenuate accumbens dopamine release to rewards but not their predictors. Nat Neurosci 16:383-385.

Wanat MJ, Kuhnen CM, Phillips PEM (2010) Delays conferred by escalating costs modulate dopamine release to rewards but not their predictors. J Neurosci 30:12020-12027.

Wang H, Lupica CR (2014) Release of endogenous cannabinoids from ventral tegmental area dopamine neurons and the modulation of synaptic processes. Prog Neuropsychopharmacol Biol Psychiatry $52: 24-27$.

Wang H, Treadway T, Covey DP, Cheer JF, Lupica CR (2015) CocaineInduced Endocannabinoid Mobilization in the Ventral Tegmental Area. Cell Rep 12:1997-2008.

Yohn SE, Collins SL, Contreras-Mora HM, Errante EL, Rowland MA, Correa M, Salamone JD (2016) Not all antidepressants are created equal: differential effects of monoamine uptake inhibitors on effort-related choice behavior. Neuropsychopharmacology 41:686-694. 